\title{
POLISH-ENGLISH BASIC DICTIONARY OF ARCHAEOLOGICAL TERMINOLOGY OF MODERN TOBACCO PIPE FINDINGS WITH ELEMENTS OF PIPE PRODUCTION AND TOBACCO SMOKING IN THE MODERN PERIOD
}

\begin{abstract}
The aim of this work is to present the translation of professional terminology concerning tobacco pipes acquired by excavation methods, supplementing the already existing term base of concepts with a Polish-English translation, important in the context of the current research on this subject. This dictionary is a translation of the basic terms used in English-language works (also used in works of researchers from Central and Eastern Europe) concerning descriptions of tobacco pipe remains (based on finds of stub-stemmed pipes, one-piece clay pipes and porcelain pipes) in archaeological research, the production of such paraphernalia and elements of history of tobacco smoking, which were additionally supplemented with terms currently used in Polish archaeological literature. This dictionary should be treated as a contribution to further work on standardisation of European archaeological terminology, which should provide an aid for both specialists and enthusiasts reaching for professional literature on antique pipes.
\end{abstract}

Keywords: tobacco pipe, archaeological terminology, dictionary, history of tobacco smoking, stub-stemmed pipe, one-piece clay pipe

Received: 24.05.2021 Revised: 17.06.2021 Accepted: 29.06.2021

Citation: Puziuk J. 2021. Polish-English Basic Dictionary of Archaeological Terminology of Modern Tobacco Pipe Findings with Elements of Pipe Production and Tobacco Smoking in the Modern Period. "Fasciculi Archaeologiae Historicae" 34, 131-137, DOI 10.23858/FAH34.2021.009.

\section{Introduction}

In current archaeological research in Poland there are various categories of finds from the modern period. Some of frequently identified are tobacco smoking accessories made of clay or porcelain. ${ }^{1}$ The current state of research on tobacco pipes in the context of Polish archaeology is based on diverse and disordered terminology which includes e.g. terms borrowed from abroad and concepts from the nomenclature used in collector communities. This problem is not only related to domestic

*Archaeological Museum in Kraków, Department of Mediaeval and Modern Times Archaeology; https://orcid.org/0000-00023055-9898;.jpuziuk@ma.krakow.pl

${ }^{1}$ Cf. Żurowski 1951; Witkowska 1989; Mikłaszewicz 1993; Zimmermann 1994; Meyza 1997; Witkowska 1998; Meyza 1999; Dąbal 2010; Bis 2011; Kwiatkowski 2011; Szewczyk-Wojtasiewicz 2017; Puziuk and Szewczyk-Wojtasiewicz 2018; Papaj 2020. research; it has already been noticed by some Central European archaeologists who initiated, in the German journal "Knasterkopf", the creation of a database of basic terms used in the study of antique pipes and methods of their production. They also translated it into various languages. ${ }^{2}$ Thanks to these activities, it was possible to select the most important terms used by archaeologists in the process of describing and handling pipes acquired by excavation methods trying at the same time to unify foreign-language nomenclature for analysed items. The results of that work included a Polish-German dictionary prepared by Ralf Kluttig-Altmann and Martin Kügler (published in 2005). These paper was

\footnotetext{
${ }^{2}$ Kluttig-Altmann et al. 2003; Kluttig-Altmann and Kügler 2004a; Kluttig-Altmann and Kügler 2004b; Kluttig-Altmann and Kügler 2005a; Kluttig-Altmann and Kügler 2005b; KluttigAltmann and Vyšohlíd 2009.
} 
intensified especially by numerous finds in Germany of one-piece pipes made in Zborowskie and Rościn which were former Prussian manufactories the remains of which are now within the Polish borders. ${ }^{3}$ This dictionary has so far been the only attempt at translating into Polish the basic terms developed in archaeological studies on tobacco pipes.

The present work is a continuation of the attempts to translate professional terminology concerning tobacco pipes acquired by excavation methods. It also complements the existing term base with a Polish-English translation, important in the context of the current research on this subject. The dictionary presented is the result of work conducted on the antique pipes at the Archaeological Museum in Kraków (as a part of the research programme Pipes and other tobacco smoking accessories in the collection of the Archaeological Museum in Kraków), which also involved an analysis of a number of English-language publications confirming the author's conviction that there is a need for such a lexical resource.

This dictionary is a translation of the basic terms used in English-language works (also used in works of researchers from Central and Eastern Europe) concerning descriptions of tobacco pipe remains (based on a finds of stub-stemmed pipes, one-piece clay pipes and porcelain pipes) in archaeological research, the production of such paraphernalia and elements of history of tobacco smoking, which were additionally supplemented with terms currently used in Polish archaeological literature. It should be noted that the vocabulary presented does not constitute a lexicon explaining the terminology of the issue touched upon here, which has already been raised more than once in the Polish archaeological literature and will not be further discussed here. ${ }^{4}$ However, in order to provide a more effective orientation and understanding of the research matter touched upon, some terms have been briefly characterised (texts in brackets at the Polish entries) and referred to relevant graphic diagrams.

This dictionary should be treated as a small contribution to further work on standardisation of European archaeological terminology, which should provide an aid for both specialists and enthusiasts reaching for professional literature on antique pipes.

\section{Polish-English dictionary}

\section{A}

agat (narzędzie kamienne z drewnianą rączką służące

do polerowania powierzchni fajki) $=$ agate tool angoba (rodzaj powłoki z rozrzedzonej gliny pokrywającej powierzchnię fajki) $=$ slip asortyment $($ fajczarski) $=$ range

\section{B}

beczkowaty (kształt, który może mieć cylinder/kominek fajki) = barrel shaped; $\sim$ cauldron-shaped brzusiec (element główki fajki) = belly (Fig. 2:2)

\section{C}

cech fajkarzy $=$ pipemaker's guild

cybuch fajki złożonej-lulki = stub-stemmed pipe stem; $\sim$ shank (Fig. 1:2)

cybuch $=$ stem $($ Fig. 1:1-3)

cylinder $(=$ kominek; element główki fajki $)=$ headbowl (Fig. 2:1-3)

czibuk $=$ chibouk $($ Fig. 4:5)

\section{D}

deska $($ do suszenia fajek $)=$ dozening board; $\sim$ drying tray długość cybucha $=$ stem length

${ }^{3}$ Kluttig-Altmann and Kügler 2005a, 104. drewniany cybuch fajki (z ustnikiem) = wood stem; $\sim$ wooden stem

drut (narzędzie do przebijania kanału dymowego fajki) $=$ piercing rod; $\sim$ wire

\section{F}

fajans $=$ faience

fajczarstwo $=$ the art of pipe smoking

fajka $=$ tobacco pipe

fajka austro-węgierska= Austro-Hungarian pipe (Fig. 4:4)

fajka hurtowa $=$ communal pipe

fajka Jakuba = Jacob pipe (Fig. 4:2)

fajka jednorodna $=$ one-piece clay pipe; $\sim$ western white clay pipe (Fig. 1:1)

fajka Jonasza $=$ Sir Walter Raleigh pipe (Fig. 4:1)

fajka kawiarniana $=$ Café-type pipe; $\sim$ Coffee-house pipe (Fig. 4:3)

fajka odlana $=$ casted pipe; $\sim$ slip-cast pipe

fajka pielgrzymia $=$ pilgrim's pipe

fajka porcelanowa (= fajka złożona z główką porcelanową $)$ porcelain pipe; $\sim$ three-piece porcelain pipe (Fig.1:3)

fajka porcelanowa dwukomorowa $=$ two chamber porcelain pipe

\footnotetext{
${ }^{4}$ Cf. Żurowski 1951, 39-42; Bis 2009, 230-237; Bis 2011, 5457; Puziuk 2015, 52; Szewczyk-Wojtasiewicz 2017, $27-34$.
} 
fajka reklamowa $=$ advertising pipe; $\sim$ pipe bearing an advertisement

fajka turecka $=$ turkish pipe; $\sim$ eastern chibouk

fajka upominek = commemorative pipe

fajka wodna $($ szisza $)=$ hookah

fajka $\mathrm{z}$ długim cybuchem = long-stemmed pipe

fajka $\mathrm{z}$ herbem $=$ heraldic pipe

fajka $\mathrm{z}$ noskiem $=$ pipe with heel or spur

fajka $\mathrm{z}$ portretem $=$ portrait pipe

fajka $\mathrm{z}$ sepiolitu $(=\mathrm{z}$ pianki morskiej $)=$ meerschaum pipe

fajka żebrowana $=$ ribbed pipe

fajka złożona-lulka $=$ stub-stemmed pipe; $\sim$ socketed pipe (Fig. 1:2)

fajka żołnierska (=,fajki rezerwisty”) = armorial pipe

fajkarstwo = pipe craft; $\sim$ pipe industry

fasetowany $=$ faceted

fidybus (zwitek papieru służący do zapalania fajki) $=$ fidibus

figurka $\mathrm{z}$ gliny fajczarskiej $=$ pipe-clay figure

forma $($ do wyciskania fajek $)=$ mould; $\sim$ pipe mould; mosiężna $=$ brass mould

forma gipsowa $($ do wyciskania fajek $)=$ plaster $($ or Paris $)$ mould

formowanie (półwyrobu, w formie do wyciskania fajki)

$=$ to mould; $\sim$ moulding

fragment fajki $=$ pipe fragment

futerał $($ na fajkę $)=$ pipe case

\section{G}

glazura (= polewa, szkliwo; rodzaj powłoki pokrywającej powierzchnię fajki) $=$ glaze

glazurowany $=$ glazed

glina (surowiec do produkcji fajek) = clay pipe; chuda glina $=$ lean clay

główka (fajki) z cylindrem/kominkiem w kształcie okrągłym $=$ rounded bowl

główka (fajki) z cylindrem/kominkiem w kształcie dys-

$\mathrm{ku}($ lub osadzonym $\mathrm{w}$ dysku $)=$ disc-based bowl

główka (fajki) z cylindrem/kominkiem w kształcie kielicha kwiatu = lily-shaped bowl

główka (fajki) z półokrągłym dnem = heelless bowl główka fajki $=$ pipe bowl

główka fajki jednorodnej = one-piece pipe bowl (Fig. 2:1) główka fajki porcelanowej $=$ porcelain pipe bowl (Fig. 2:3) główka fajki złożonej-lulki = stub-stemmed pipe bowl

(Fig. 2:2)

\section{H}

herb (zdobienie na główce lub sygnatura w kształcie tarczy herbowej) $=$ coat of arms

\section{I}

inicjały (producenta fajek) $=$ initials; $\sim$ letters inskrypcja na cybuchu = inscription on the stem

\section{$\mathbf{J}$}

jajowaty (kształt główki fajki) = egg-shaped

\section{$\mathbf{K}$}

kamionka $=$ fine earthenware; $\sim$ stoneware

kanelura (zdobienie główki fajki) = fluting

kapturek (metalowe, najczęściej mosiężne wieczko zakrywające cylinder/kominek główki fajki) = lid; $\sim$ cap (Fig. 1:3 and 3)

kartusz (zdobienie główki fajki) - cartouche

komora napychowa (element główki fajki) $=$ firing chamber; tobacco chamber (Fig. 2:1-3)

kondensat tytoniowy = tobacco condensate; $\sim$ juice

kopalnia gliny $=$ clay mine; $\sim$ pit

kryza (metalowe, najczęściej mosiężne okucie obrzeża główki fajki) $=$ rim (Fig. 3)

kryza główki (obrzeża cylindra/kominka) $=$ rim of the bowl

kształt główki $=$ shape of the bowl

\section{$\mathbf{L}$}

lakierowany $=$ varnished

lejkowaty (kształt główki fajki) = funnel-shaped

lilia (zdobienie główki fajki) $=$ fleur-de-lis

lilia $\mathrm{w}$ rąbie (zdobienie główki fajki) = diamond enclosing fleur-de-lis

lulka $=$ stub-stemmed pipe; $\sim$ socketed pipe (Fig. 1:2)

muszelkowaty $($ kształt $)=\stackrel{\text { M }}{\text { scalloped }}$

\section{$\mathbf{N}$}

naczynie na żar (lub popiół) = brazier

narzędzie do wygładzania (powierzchni fajki) = finish-

ing tool; $\sim$ smoothing tool

narzędzie do formowania otworu (napychu) w główce

fajki $=$ stopper

nosek $(=$ ostroga $)=$ heel $($ Fig. $2: 1,3)$

\section{$\mathbf{O}$}

obrzeże (= krawędź; element cybucha/kominka lub szyjki) $=$ edge (Fig. 2:1-3)

odpadki produkcyjne (przy wypalaniu fajek) = kiln waste;

$\sim$ pipe wasters; $\sim$ spoilage

okrągły (kształt, który może mieć cylinder/kominek

fajki) $=$ bulbous

ornament dookolny cybucha $=$ rolled-on ornamentation ornament łuskowy $=$ scale ornament

ornament na brzegu (główki fajki) = milling

ornament na obrzeżu/krawędzi $=$ denticulated motif

ornament plecionkowy $=$ basket-weave design

ostroga $(=$ nosek $)=$ heel

otwór $=$ mouth

otwór paleniska pieca (fajkarskiego) = stoke hole 
otwór tulejki (szyjki główki fajki) = shank opening owoidalny (kształt główki fajki) = ovoid shaped ozdoba $\mathrm{w}$ formie reliefu $=$ moulded decoration

\section{$\mathbf{P}$}

palenisko pieca (fajkarskiego $)=$ furnace

palmeta (zdobienie główki fajki) $=$ palmette

piec do wypalania (fajek) $=$ kiln

piec z małymi pojemnikami $=$ muffle kiln

pięta (płaska powierzchnia noska fajki jednorodnej lub porcelanowej $)=\operatorname{spur}($ Fig. $2: 1)$

plecionka (zdobienie na fajkach) = basket-weave design podwójnie stożkowaty = double-conical; $\sim$ bi-conical

pojemnik do wypalania fajek = fireclay box; $\sim$ saggar;

$\sim$ sagger

polerowany (= wyświecany; zdobienie główki fajki) $=$ polished

powierzchnia $($ fajki $)=$ surface

półfabrykat $=$ blank

prasa (urządzenie mechaniczne służące do wyrobu fajek $)=$ gin press

próbka fajki (półprodukt wyciągnięty z pieca w celu obserwacji postępów wypalania) $=$ pipe taken out of the kiln with tongs during firing to test the progress of firing

przebijanie kanału dymowego $=$ piercing

przekrój przewodu dymnego = stem bore; $\sim$ hole diameter; hole bore

przewód dymny $=$ stem hole (Fig. 2:1-3)

przybory do palenia $=$ smoking equipment

przygotowanie gliny (masy glinianej do produkcji fajek) $=$ clay preparation

przyrządy pomocnicze przy wypalaniu (fajek) $=$ kiln furniture

\section{$\mathbf{R}$}

rozmiar główki fajki $=$ bowl size

rozszerzający się otwór główki (cylindra/kominka)

= flared mouth

rozwój kształtu główki = bowl development

rzeźbiarz fajek = pipe carver

\section{$\mathbf{S}$}

sepiolit (= pianka morska; surowiec do produkcji fajek)

$=$ meerschaum

spód fajki (główki) = base of bowl; bottom

stępka (fragment szyjki znajdujący się pod nasadą cylindra) $=$ keel

stosowanie tytoniu (czerpanie przyjemności z nałogu tytoniowego) $=$ consumption of tobacco; enjoyment of tobacco

stożkowaty (kształt główki fajki lub zbiornika na kondensat) $=$ cone shaped

strona czołowa główki fajki = back of the bowl (Fig. 1:2) strona przednia główki fajki $=$ front of the bowl

(Fig. 1:2)

sygnatura $=$ mark; maker's initials; $\sim$ stamp (Fig. 1:1)

sygnatura cyfrowa $=$ mark depicting a numeral; $\sim$ nu-

merical mark

sygnatura figuralna $=$ pictorial mark; $\sim$ picture patterned

mark

sygnatura formy $=$ mould identification mark

sygnatura literowa $=$ mark depicting initials; $\sim$ letter mark

sygnatura na boku noska $=$ mark on side of spur

sygnatura na cybuchu $=$ stem stamp

sygnatura na nosku $=$ mark on spur; $\sim$ spur mark; $\sim$ heel

mark

sygnatura na nosku $($ pięcie $)=$ mark on spur; $\sim$ spur

mark; heel mark

sygnatura na spodzie $=$ mark on the base of bowl

sygnatura na stronie czołowej $($ główki fajki $)=$ mark on

the back of the bowl

sygnatura podrobiona (imitacja oryginalnej sygnatury

wykonana przez fałszerza fajek) $=$ pirated mark

sygnatura $\mathrm{w}$ formie reliefu $=$ moulded mark; $\sim$ relief mark sygnowanie $($ fajki) $=$ marking

sześciokątny (kształt cylindra fajki) = hexagonal; $\sim$ six

sided

szew $=$ mould line; $\sim$ seam

szyjka (element główki fajki) $=$ neck (Fig. 2:2-3)

\section{Ś}

ścianka główki fajki $=$ bowl wall

ślady użytkowania (fajki) = signs of use

średnica cybucha $=$ diameter of stem

\section{T}

tabaka $=$ snuff

tulejka (element główki fajki) $=$ mortise (Fig. 2:2)

\section{$\mathbf{U}$}

ubijak (przyrząd służący do ubijania tytoniu podczas palenia) $=$ pipe tamper

układanie fajek glinianych w pojemniku do wypalania

$=$ to pack (or place) pipes in a sagger

uprawa tytoniu $=$ tobacco growing

ustnik (element fajki jednorodnej lub złożonej) = mouth-

piece (Fig. 1:1-3)

używka $=$ stimulant

\section{W}

wałek (element główki fajki złożonej-lulki) = ring

(Fig. 2:2)

warsztat fajkarski (produkujący fajki, zakład rzemieślniczy) $=$ pipemaker's workshop

właściciel zakładu (fajkarskiego) = factory owner wygładzający (rzemieślnik wygładzający powierzchnię fajki) $=$ finisher; $\sim$ trimmer 
wygładzanie $($ powierzchni fajki) $=$ finishing; $\sim$ trimming wypalanie $($ fajek $)=$ to fire

wypolerowanie (powierzchni fajki) = to burnish; to

smooth

wytwórca fajek (również mistrz fajkarski) = pipemaker wytwórca form (do wyciskania fajek) = mouldmaker wytwórnia fajek (fabryka) = pipe factory; $\sim$ pipe works

wzdłuż cybucha $=$ along the stem; longitudinal

\section{$\mathbf{Z}$}

zakaz palenia (restrykcje, które spotykały palaczy) $=$ ban on smoking

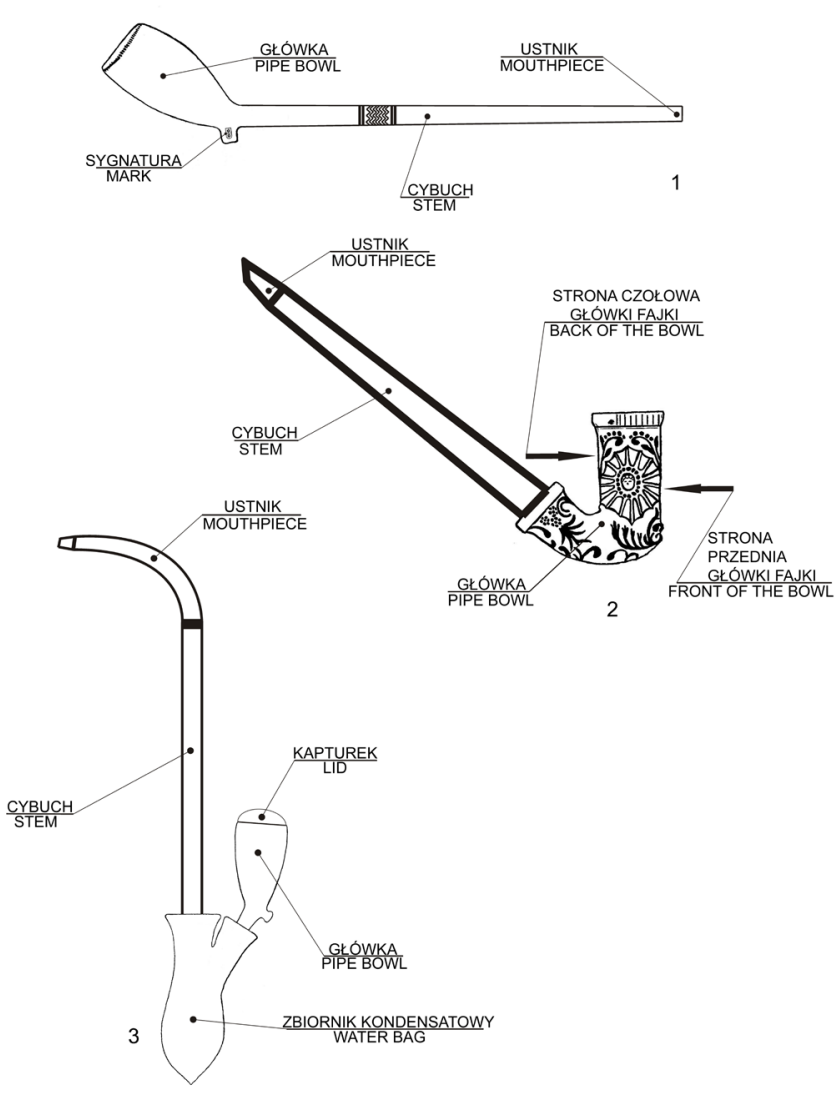

Fig. 1. Schematic reconstruction: 1 - one-piece clay pipe; 2 - stub-stemmed pipe; 3 - porcelain pipe. Graphic design by J. Puziuk. zaokrąglanie brzegów główki fajki $=$ to round off the rim of the bowl

zażywanie tabaki $=$ snuff taking

zbiornik na kondensat $(=$ osadnik; element fajki złożonej

z główką porcelanową) $=$ water bag (Fig. 1:3)

zespół ornamentów (grupa motywów zdobniczych na powierzchni fajki) $=$ band of ornament; $\sim$ ornamental band

zwijanie gliny $w$ wałki $=$ to roll on

\section{$\dot{\mathbf{Z}}$}

żłobienie (powierzchni fajki) $=$ hollow

żucie tytoniu $=$ chewing tobacco
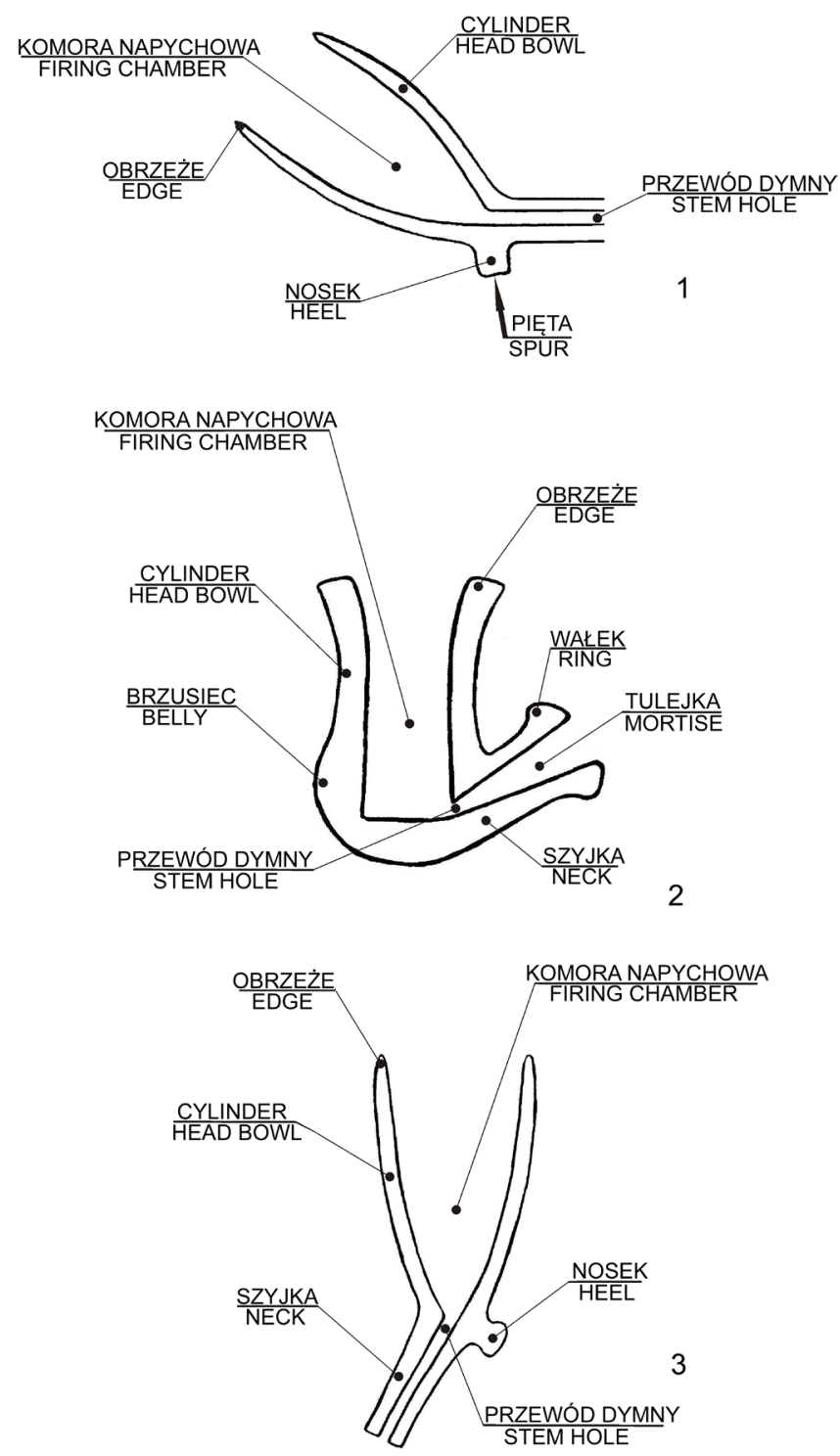

Fig. 2. Cross-sections with construction elements: 1 - one-piece pipe bowl; 2 - stub-stemmed pipe bowl; 3 - porcelain pipe bowl. Graphic design by J. Puziuk 

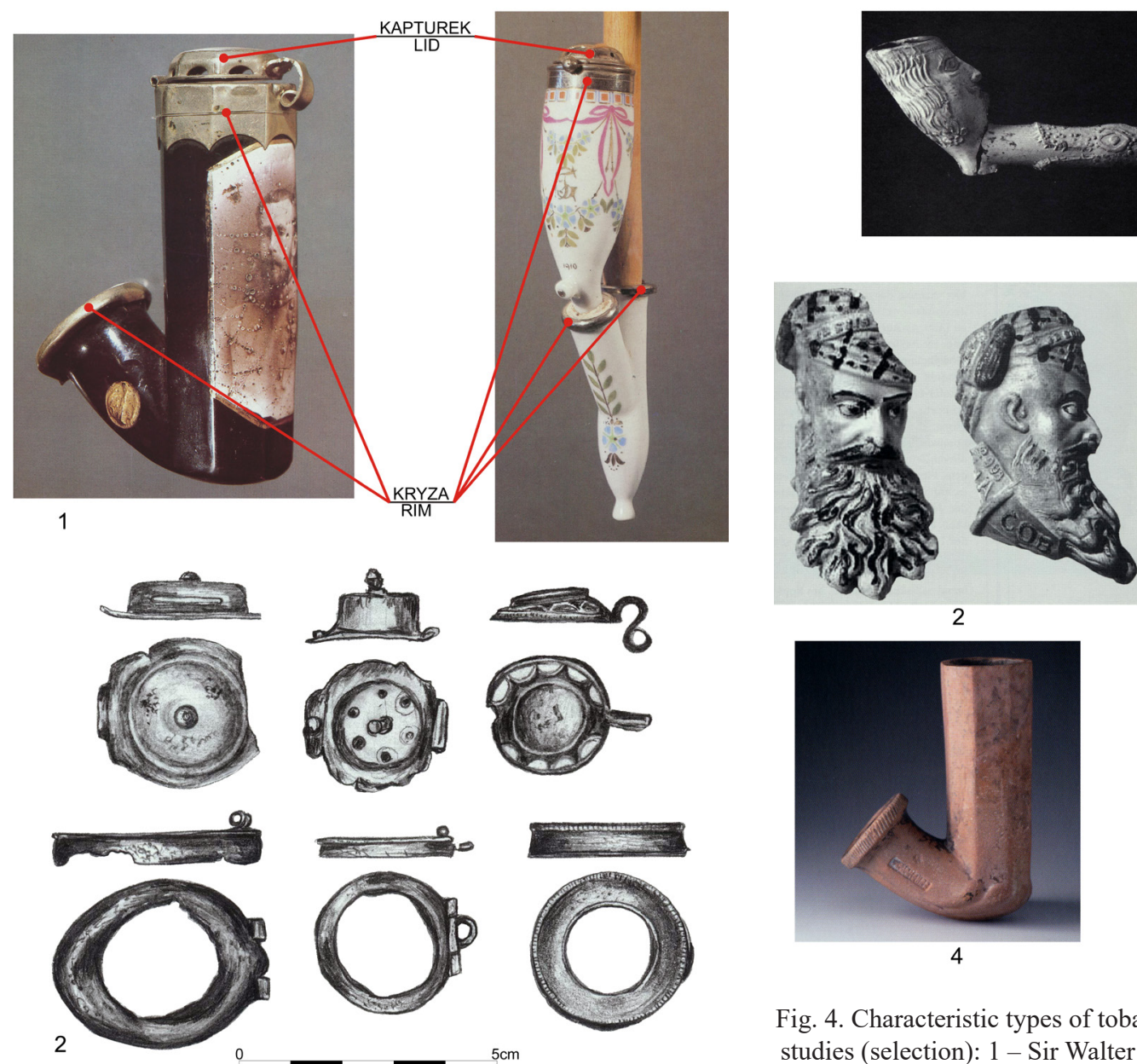

2

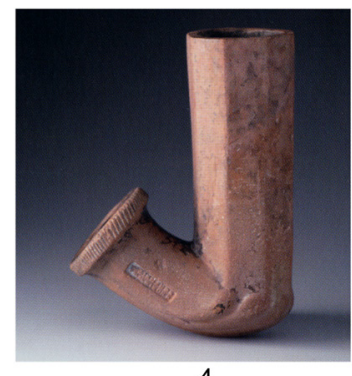

4

Fig. 4. Characteristic types of tobacco pipes used in archaeological studies (selection): 1 - Sir Walter Raleigh pipe. After Libert 1984, 42; 2 - Jacob pipe. After Raphaël and Kügler 2005, 139;

3 - Café-type pipe. After Szewczyk-Wojtasiewicz 2011, 48;

4 - Austro-Hungarian pipe. After Szewczyk-Wojtasiewicz 2011, 40; 5 - chibouk. After Batchvarov 2014, Fig. 10.

1984, 62, 72; 2 - examples of such archaeological finds from Wawel

Hill in Kraków. After Szewczyk-Wojtasiewicz 2017, 428, 432.

\section{References}

Batchvarov K. 2014. Clay Pipes and Smoking Paraphernalia from the Kitten Shipwreck, a Nineteenth-Century Black Sea Merchantman. "International Journal of Historical Archaeology" 18, 1-19.

Bis M. 2009. O glinianych lulkach w dawnej Rzeczypospolitej. "Kwartalnik Historii Kultury Materialnej” 57(2), 229-242.

Bis M. 2011. Lulki z wykopalisk na zamku w Tykocinie z XVII-XIX w. "Kwartalnik Historii Kultury Materialnej" 59(1), 53-88.

Dąbal J. 2010. Jednorodne fajki gliniane z manufaktury w Rościnie pozyskane na stanowisku przy ul. Szafera w Gdańsku. Próba interpretacji źródet w kontekście zmian społeczno-ekonomicznych w okresie industrialnym. In: B. Bobowski (ed.), Archeologia Dolnego Miasta w Gdańsku. Pótnocno-zachodnia część kwartału ulic Dlugie Ogrody, Szafarnia, Angielska Grobla, Św. Barbary. Gdańsk, 73-86.

Holčík Š. 1984. Fajky. Knižnica priatel’ov výtvarného umenia 34. Bratislava.

Kluttig-Altmann R., Kügler M. 2004a. Internationale Terminologie der Tonpfeifenforschung. Teil II: Niederländisch - Deutsch. "Knasterkopf. Fachzeitschrift für Tonpfeifen und historischen Tabakgenuss" 17, 67-69.

Kluttig-Altmann R., Kügler M. 2004b. Internationale Terminologie der Tonpfeifenforschung. Teil III: Französisch - Deutsch. "Knasterkopf. Fachzeitschrift für Tonpfeifen und historischen Tabakgenuss" 17, 70-73.

Kluttig-Altmann R., Kügler M. 2005a. Internationale Terminologie der Tonpfeifenforschung. Teil IV: PolnischDeutsch. "Knasterkopf. Fachzeitschrift für Tonpfeifen und historischen Tabakgenuss" 18, 104-106.

Kluttig-Altmann R., Kügler M. 2005b. Internationale Terminologie der Tonpfeifenforschung. Teil V: UngarischDeutsch. "Knasterkopf. Fachzeitschrift für Tonpfeifen und historischen Tabakgenuss" 18, 107-109. 
Kluttig-Altmann R., Kügler M., Toms H. 2003. Internationale Terminologie der Tonpfeifenforschung. Teil I: Englisch-Deutsch. "Knasterkopf. Fachzeitschrift für Tonpfeifen und historischen Tabakgenuss" 16, 149-152.

Kluttig-Altmann R., Vyšohlíd M. 2009. Internationale Terminologie der Tonpfeifenforschung. Teil VI: Tschechisch-Deutsch. "Knasterkopf. Fachzeitschrift für Tonpfeifen und historischen Tabakgenuss" 20, 80-84.

Kwiatkowski K. 2011. Jednorodne fajki gliniane z badań kwartału staromiejskiego w Stargardzie. "Materiały Zachodniopomorskie" NS 6/7, 357-382.

Libert L. 1984. Von Tabak, Dosen und Pfeifen. Leipzig.

Meyza K. 1997. Fajki gliniane z XVII w. i pierwszej połowy XVIII w. z badań archeologicznych Zamku Królewskiego i Starego Miasta w Warszawie. "Almanach Muzealny” 1, 31-44.

Meyza K. 1999. Fajki gliniane z drugiej połowy XVIII i pierwszej połowy XIX w z badań archeologicznych Zamku Królewskiego i miasta Warszawy. "Almanach Muzealny" 2, 5-21.

Mikłaszewicz D. 1993. Fajki z wraków zalegających na dnie Zatoki Gdańskiej. "Pomorania Antiqua" 15, 265-299.

Papaj B. 2020. Analiza materiału ruchomego pozyskanego w trakcie badań archeologicznych przeprowadzonych w 2013 i 2014 roku w miejscu funkcjonowania XVIII-wiecznej fabryki fajek glinianych w miejscowości Zborowskie (woj. ślaskie, pow. lubliniecki). In: B. Papaj (ed.), Ślaska fabryka fajek glinianych w Zborowskiem. Wyniki badań, studia, konteksty. Chorzów, 297-465.

Puziuk J. 2015. Nowożytne fajki z badań archeologicznych w Krakowie. "Materiały Archeologiczne" 40, 51-85.

Puziuk J., Szewczyk-Wojtasiewicz M. 2018. Nowożytne akcesoria do palenia tytoniu z badań archeologicznych na Zamku Krzyżtopór w Ujeździe. "Rocznik Muzeum Narodowego w Kielcach” 33, 175-195.

Szewczyk-Wojtasiewicz M. 2011. Fajki w zbiorach Zamku Królewskiego na Wawelu. Kraków.

Szewczyk-Wojtasiewicz M. 2017. Fajki z badań archeologicznych w zbiorach Zamku Królewskiego na Wawelu. Kraków.

Witkowska T. 1989. Manufaktura fajek glinianych z Rościna, gmina Myślibórz. "Materiały Zachodniopomorskie" 35/36 (1989-1990), 283-301.

Witkowska T. 1998. Fajki z badań archeologicznych na placu Dominikańskim we Wrocławiu. "Silesia Antiqua" 39, 283-336.

Zimmermann E. 1994. Fajki gliniane. Gdańsk.

Żurowski T. 1951. Fajki gliniane (Z badań archeologicznych). "Ochrona Zabytków” 4/1-2 (12-13), 39-57. 
